\title{
Polyelectrolyte Surface Interface for Single- Molecule Fluorescence Studies of DNA Polymerase
}

BioTechniques 34:505-510 (March 2003)

\begin{abstract}
We report the use of polyelectrolyte multilayers in a stable robust surface chemistry for specific anchoring of DNA to glass. The nonspecific binding of fluorescently tagged nucleotides is suppressed down to the single-molecule level, and DNA polymerase is active on the anchored DNA template. This surface-chemistry platform can be used for single-molecule studies of DNA and DNA polymerase and may be more broadly applicable for other situations in which it is important to have specific biomolecular surface chemistry with extremely low nonspecific binding.
\end{abstract}

\section{INTRODUCTION}

Since most enzymes have evolved to function in the solution phase, it can be challenging to design surface chemistries that allow specific anchoring of enzymes at solid-liquid phase interfaces while retaining the activity of the enzyme. With the development of new biophysical techniques that take advantage of single-molecule fluorescent imaging technology, there has been a renewed interest in developing methods for specific binding of enzymes and other biological molecules to planar glass surfaces, thus allowing optical access (1). Much of the existing literature has focused on developing techniques to anchor fluorescently labeled proteins to the surface and monitoring the activity of the enzyme by conformation-induced changes in fluorescence (2-4). In some cases, it is possible to find a substrate for the enzyme of interest that gets catalyzed from a nonfluorescent to a fluorescent form, thus allowing the use of an unlabeled enzyme and relatively high concentrations of the substrate in solution (5). However, in the most general situation, one would like to study unmodified enzymes whose activity does not change the fluorescence of the substrate. In those cases, the surface chemistry becomes challenging because one must simultaneously satisfy several constraints - the enzyme must be specifically anchored and active, and the surface must resist nonspecific binding of a relatively high concentration of fluorescently labeled substrate molecules.

Most single-molecule enzyme-anchoring surface chemistries, such as PEG grafts (2) or nickel-derivatized surfaces (3), are designed to anchor an active enzyme but do not address the issue of nonspecific binding of labeled substrate molecules. In cases where the enzyme can be modified and two fluorescent molecules are used, fluorescence resonance energy transfer has been utilized to decrease noise from background fluorescence (3). Here we report the use of polyelectrolyte multilayers to tune the charge density on the surface to repel labeled substrate molecules selectively. The advantage of this method is that it achieves an extremely low density of nonspecifically bound substrate molecules, thus enabling the use of a single fluorescence channel and avoiding the need for modification of the enzyme of interest.

Polyelectrolytes are polymers whose chains contain charged functional groups. Some examples are polystyrene sulphonate, polylysine, polyglutamic acid, polyacrylic acid (PAcr), polyethyleneimine (PEI), and poly(allylamine) (PAll). Decher et al. $(6,7)$ have built polyelectrolyte multilayers by sequential deposition of polyanions and polycations and have investigated their structure and growth process. Polyelectrolyte multilayers are an excellent platform for surface chemistry and have been used to study DNA-PAll composites (8), layers of a charged virus (9), streptavidin (10), and various other proteins (11). Chluba et al. (12) used polyelectrolyte multilayers to build a biologically active hormone layer for implant and tissue engineering. Stroock et al. (13) adsorbed polyelectrolyte layers in microchannels to pattern surface charge and electro-osmotic flow.

We used a polyelectrolyte multilayer structure to specifically anchor the DNA template used by DNA polymerase. A polyelectrolyte multilayer is created, and the final layer is used as a function- 


\section{Short Technical Reports}

alization target for the covalent attachment of biotin. This allows anchoring of DNA through biotin-streptavidin specific bonding, instead of nonspecific deposition in a dense layer (8). The polyelectrolyte multilayer is constructed with a negative ionic final layer that repels negatively charged, fluorophore-tagged nucleotide triphosphates. We found that nonspecific binding is suppressed sufficiently to enable fluorescence studies of enzymatic processes involving single DNA molecules. Thus, we have developed a platform that is suited for singlemolecule studies of DNA and DNA polymerase, enabling applications such as single-molecule DNA sequencing, bulk DNA sequencing through synthesis, DNA hybridization microarrays, and enzymological research at the single-molecule level.

\section{MATERIALS AND METHODS}

Corning microscope slides $(3 \times 1$ in $)$ and VWR Micro Cover Glasses no. 1 coverslips $(25 \times 25$ or $22 \times 22 \mathrm{~mm})$ are cleaned using a version of the RCA protocol (14) and stored in HP water (18 M $\Omega \mathrm{m}, 0.2 \mu \mathrm{m}$ filtered).

PEI from Sigma (St. Louis, MO, USA) (or PAll from Aldrich Chemical, Milwaukee, WI, USA) and PAcr from Aldrich are dissolved at $2 \mathrm{mg} / \mathrm{mL}$ in $\mathrm{HP}$ water. The solutions are adjusted to pH 8.0 using $\mathrm{NaOH}$ and $\mathrm{HCl}$. This $\mathrm{pH}$ ensures that both components have their functional groups charged (dissociated carboxyl and protonated amino groups, respectively). The polyelectrolyte solutions are passed through a $0.22-\mu \mathrm{m}$ filter to remove dust residue from the solid phase.

The RCA-cleaned glass is loaded into microslide mailers from Thomas Scientific and then immersed in solutions of the positive (PEI or PAll) and negative (PAcr) polyelectrolytes according to the scheme +/wash/-/wash/ +/wash/-/wash. Each polyelectrolyte step is 10 min of immersion, whereas a wash step is thorough rinsing with HP water. Polyelectrolyte multilayer glass is stored in HP water and retains surface charge for at least several months. We have observed that PEI and PAll behave essentially the same for purposes of the presented surface chemistry but that PEI is generally more efficient to handle experimentally.

Polyelectrolyte multilayer glass is biotinylated using the EZ-Link ${ }^{\mathrm{TM}}$ kit from Pierce Chemical (Rockford, IL, USA). Fifty millimolar 1-[3-(Dimethylamino)propyl]-3-ethylcarbodiimide hydrochloride (EDC; Aldrich Chemical) is freshly dissolved in $10 \mathrm{mM} \mathrm{2-[N-}$ Morpholino]ethanesulfonic acid buffer (MESb), pH 5.5. The EDC solution is filtered to remove any dust residues from the solid phase. Biotin-LC-PEOAmine from the kit $(50 \mathrm{mM})$ in $\mathrm{MESb}$ is mixed with the EDC solution at 1:1, and the result is diluted to $5 \mathrm{mM}$ final concentration of each in MESb. This solution is pipetted onto the polyelectrolyte multilayer surface to biotinylate it. After incubation for $30 \mathrm{~min}$ in a humid chamber, the unreacted excess is washed away with MESb and then with Trisb (10 mM Tris, $10 \mathrm{mM} \mathrm{NaCl}$, pH 8.0).

Streptavidin-Plus ${ }^{\mathrm{TM}}$ from Prozyme (San Leandro, CA, USA) $(40 \mu \mathrm{L}, 0.1$ $\mathrm{mg} / \mathrm{mL}$ in Trisb) is pipetted onto the biotinylated polyelectrolyte multilayer surface. After a 30-min incubation in a humid chamber, the unreacted excess is washed off with Trisb. Then, $40 \mu \mathrm{L}$ DNA at $0.1 \mu \mathrm{M}$ for bulk experiments or $0.1 \mathrm{nM}$ for single-molecule experiments in TrisMg (10 mM Tris, $10 \mathrm{mM}$ $\mathrm{NaCl}, 100 \mathrm{mM} \mathrm{MgCl} 2, \mathrm{pH} 8.0$ ) is deposited on the surface. After $30 \mathrm{~min}$ of DNA deposition in a humid chamber, the unreacted excess is washed off with Trisb. This completes the procedure.

Mu50, the DNA used in the experiments, was a biotinylated 50-mer (Biotin-5'-CTCCAGCGTGTTTTATCTCTGCGAGCATAATGCCTGCGTCATCCGCCAGC-3') annealed to a 14mer primer (5'-GCTGGCGGATGACG- $3^{\prime}$ ), both from Operon Technologies (Alameda, CA, USA). Its sequence was taken from the Lambda phage genome and was selected for its unlikelihood to form hairpins and dimers. The DNA experiments also made use of dATP-Lissamine (A-Lis) from NEN Life Science Products (Boston, MA, USA); dCTP-Cy3 (C-Cy3) from Amersham Biosciences (Piscataway, NJ, USA); dATP-Tetramethylrhodamine (A-TMR), dATP-Texas Red (A-Tex) from Molecular Probes (Eugene, OR, USA); and non-tagged nucleotide triphosphates from Roche Applied Sci- ence (Mannheim, Germany). Klenow Fragment ( $3^{\prime}-5^{\prime}$ exo-) DNA polymerase (Klenow exo-) from New England Biolabs (Beverly, MA, USA) was used at $50 \mathrm{U} / \mathrm{mL}$ reaction volume.

Optical observations were conducted using an inverted microscope (Olympus IX50) with a mercury lamp, an Olympus PlanApo $60 \times$ objective (N.A. 1.4), a cooled CCD camera (model ST-7I; SBIG, Santa Barbara, CA, USA), and fluorescence filter sets appropriate for the particular dyes: Fluorescein (ex D470/40, 500 DCLP, em D535/50), TMR and Cy3 (ex D540/25, dichroic 565 DCLP, em D605/55), Lissamine and Texas Red (ex D560/40, dichroic 595 DCLP, em D630/60) (all from Chroma Technology, Brattleboro, VT, USA).

\section{RESULTS AND DISCUSSION}

The initial RCA cleaning procedures leaves hydroxyl groups on the glass surface, which are deprotonated at the $\mathrm{pH}$ used here, and thus impart negative charge to the surface. While this charge can provide some electrostatic shielding against nonspecific adsorption of tagged nucleotides, the surface charge density is very low. To increase this density, we build up polyelectrolyte layers. The positively charged PEI binds electrostatically to the negatively charged glass. The negatively charged PAcr binds to PEI for the same reason. This process is repeated, and the final fourth layer of the polyelectrolyte multilayer is the negatively charged PAcr, which repels the negatively charged, fluorescently tagged nucleotides. The polymeric nature of the polyelectrolyte multilayer results in increased charge density for each adsorbed layer, allowing us to tune the charge density and to cover any inhomogeneities on the surface that might become sites for nonspecific attachment.

To examine the polyelectrolyte multilayer surface charge experimentally, we exposed positively and negatively terminated polyelectrolyte multilayer surfaces to dATP-TMR, which carries effectively three negative charges per molecule at $\mathrm{pH}$ 8.0. Thus, we expected to detect much larger fluorescent signal from positive surfaces than from negative ones because of the electrostatic in- 
Table 1. Electrostatic Effects

\begin{tabular}{|lrc|}
\hline Preparation & Signal & Uncertainty \\
\hline PEM + Trisb & 542 & 11 \\
PEM + dATP-TMR & 4121 & 475 \\
PEM(+) + Trisb & 489 & 10 \\
PEM (+) + dATP-TMR & 77238 & 2814 \\
PEM + (BLCPA + EDC) + SA-TRITC & 39256 & 455 \\
PEM + (BLCPA) + SA-TRITC & 1127 & 62 \\
PEM + (EDC) + SA-TRITC & 714 & 68 \\
PEM-Biotin + SA + Biotin-DNA-Fluorescein & 9159 & 185 \\
PEM-Biotin + Biotin-DNA-Fluorescein & 629 & 118 \\
PEM-Biotin + SA + Biotin-Fluorescein & 133550 & 5282 \\
PEM-Biotin + Biotin-Fluorescein & 622 & 12 \\
Polyelectrolyte multilayer (PEM) shielding prevents nonspecific attachment of \\
tagged nucleotides, while 97\% of streptavidin attaches specifically to biotinylated \\
polyelectrolyte multilayer surfaces. The presence of streptavidin greatly increases \\
the attachment of biotinylated DNA and fluorescein. The numbers are in counts \\
per pixel. Each pixel is 0.45 $\times$ 0.45 $\mu$ m. Abbreviations can be found in the text. \\
\hline
\end{tabular}

teractions. Accordingly, we prepared four polyelectrolyte multilayer coverslips following the general procedure from the Materials and Methods section up to the biotinylation step (using PAll). Then, two of the slips were put through an extra treatment of PAll/ wash. These slips were called $\operatorname{PEM}(+)$ to indicate that they are positively terminated, while the standard ones, PEM, are negatively terminated. Then, one slip from each group was incubated for 15 min with $1 \mu \mathrm{M}$ A-TMR in Trisb, and the other with only Trisb, as a control. All slips were then washed with Trisb and observed on the microscope. The Trisb controls established the background signal from the polyelectrolyte multilayer and glass, whereas the dATP-TMR cases gave information about the charges on the surface.

A series of several pictures was taken at different locations on each coverslip, using a dichroic filter set appropriate for TMR. For each picture, the camera signal was integrated over the central $50 \times 20 \mu \mathrm{m}$ area of the field of view to obtain an average value for the fluorescence signal in counts per pixel. The mean of these values was calculated for each series of pictures, and uncertainty was assigned as half the difference between the maximum and minimum values. The results (Table 1) showed that dATP-TMR bound 22 times more to $\operatorname{PEM}(+)$ than $\mathrm{PEM}$, as expected if the interaction were dominated by electrostatics.

In our general protocol, the biotinylated DNA is anchored specifically to the polyelectrolyte multilayer surface using biotin and streptavidin. A kit is used to link biotin to the carboxyl groups present at the PAcr surface, which is then treated with streptavidin. During the DNA anchoring step, a high concentration of $\mathrm{Mg}^{2+}$ is used to screen the electrostatic repulsion between the negatively charged DNA and the negatively charged polyelectrolyte multilayer surface. In subsequent steps, the salt concentration is reduced to reactivate the repulsive shielding.

To examine the specificity of the biotinylation step, we withheld either Biotin-LC-PEO-Amine (the kit linker) or EDC (the kit activation agent) and then tested for the presence of anchored biotin by attachment of streptavidinTRITC and fluorescence detection. We observed 40 times stronger signal (Table 1) when all the biotinylation components were present than when any were withheld. Similar tests were conducted for streptavidin deposition by withholding streptavidin and then trying to bind biotin-fluorescein or biotinylated fluorescein-tagged DNA. If the surface was treated with streptavidin, then 215 and 15 times stronger 


\section{Short Technical Reports}

Table 2. Bulk Incorporation of Fluorescently Tagged Nucleotides Inside DNA Anchored at the Surface

\begin{tabular}{|c|c|c|}
\hline Preparation & Signal & Uncertainty \\
\hline DNA + (C,T,G, A-TMR, Polymerase) & 26410 & 3728 \\
\hline $\mathrm{DNA}+(\mathrm{C}, \mathrm{T}, \mathrm{G}, \mathrm{A}-\mathrm{TMR})$ & 6291 & 225 \\
\hline DNA + (C,T,G, A-Lis, Polymerase) & 83761 & 14846 \\
\hline DNA + (C,T,G, A-Lis) & 1456 & 29 \\
\hline DNA + (C,T,G, A-Tex, Polymerase) & 13077 & 1167 \\
\hline $\mathrm{DNA}+(\mathrm{C}, \mathrm{T}, \mathrm{G}, \mathrm{A}-\mathrm{Tex})$ & 2028 & 94 \\
\hline DNA + (A, T,G, C-Cy3, Polymerase) & 24873 & 4798 \\
\hline $\mathrm{DNA}+(\mathrm{A}, \mathrm{T}, \mathrm{G}, \mathrm{C}-\mathrm{Cy} 3)$ & 495 & 15 \\
\hline \multicolumn{3}{|c|}{$\begin{array}{l}\text { In control cases, DNA polymerase was withheld; hence, the net increase in DNA } \\
\text { polymerase cases is due to incorporation. The numbers are in counts per pixel. }\end{array}$} \\
\hline
\end{tabular}

signal was observed (Table 1).

The experimental confirmation of specific anchoring of the DNA allowed us to proceed to testing for incorporation at the surface. The electrostatic shielding at the surface probably repels the unanchored end of the DNA away from the surface, which would reduce surface-promoted protein denaturation or steric hindrances that might inhibit DNA polymerase activity. We contrasted different combinations of nucleotides and fluorescent tags and compared them to controls containing no DNA polymerase. In the absence of DNA polymerase, no incorporation should happen, so any signal in that case would be a measure of nonspecific binding. The net signal increase in the DNA polymerase cases would be interpreted as the contribution from incorporation.

For this purpose, we prepared 16 coverslips with the complete chemistry (using PAll) including the anchoring of $0.1 \mu \mathrm{M}$ Mu50 in TrisMg. The coverslips were organized in eight pairs so that the degree of consistency could be measured by the difference between the results in each pair. Next, the eight pairs were organized in four groups of two pairs. EcoPol buffer $(20 \mu \mathrm{L})$ containing A-TMR, dCTP, dGTP, and dTTP, each at $1 \mu \mathrm{M}$, and $50 \mathrm{U} / \mathrm{mL}$ Klenow exo- were pipetted onto Group 1 Pair 1. Group 1 Pair 2 was treated identically, except for withholding the DNA polymerase. The same procedure was applied to the other groups, except the mixture of nucleotides was different: Group 2 (A-Texas Red, C, T, G),
Group 3 (A-Lis, C, T, G), and Group 4 (C-Cy3, A, T, G). After 40 min simultaneous incubation in a humid chamber, all slips were washed with Trisb. Fluorescence measurements were conducted using dichroic filter sets appropriate for the particular dyes. The data acquisition and analysis were conducted similarly to the previously described experiments. The results (Table 2) show up to 58 times higher signals whenever the DNA polymerase was present, which confirms incorporation inside the anchored DNA.

Single-fluorophore studies were conducted in parallel with the bulk incorporation measurements discussed above. We have previously shown that the cleaning protocols and microscope system used here allow single-molecule detection and quantification $(14,15)$. Using the quantized fluorescent signal obtained from single-molecule experiments, we are able to make absolute measurements of the surface density of proteins (15). In this case, the bulk experiments with both DNA and fluorescently labeled streptavidin showed that the surface density of streptavidin was extremely high; we estimated greater than 100 streptavidin molecules per square micron. By titrating the biotinylated DNA, it is possible to bind such a small number of molecules to the surface that they are separated by more than the diffraction limit and thus able to be visualized individually.

Accordingly, we prepared three coverslips with the complete chemistry (using PEI). The DNA used was Mu50 


\section{Short Technical Reports}

at $1 \mathrm{nM}$ in TrisMg. Then coverslip no. 1 was treated with a mixture of dATPLis, dCTP, dGTP, and dTTP each at 0.1 $\mu \mathrm{M}$ in EcoPol buffer also containing Klenow exo-. The coverslip no. 2 was prepared the same as no. 1 but without DNA polymerase. The no. 3 was prepared the same as no. 2, except for using untagged dATP instead of A-Lis. After $30 \mathrm{~min}$ incubation in a humid chamber, all slips were washed with Trisb. Observations were made with a dichroic filter set appropriate for lissamine. Samples of the resulting pictures are shown in Figures 1a (no. 1), $1 \mathrm{~b}$ (no. 2), and 1c (no. 3). Comparison of the incorporation in Figure 1a and the control in Figure 1b shows the onsurface incorporation at the singleDNA level. Comparison of the three images shows that the total contribu-
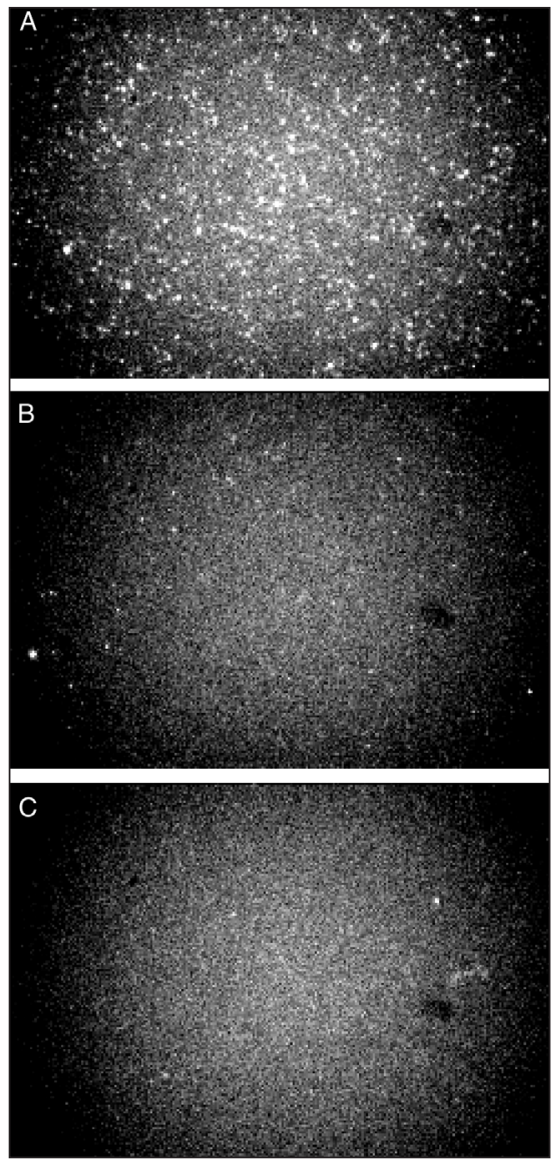

Figure 1. On-surface incorporation in anchored DNA can be visualized at the single-DNA level. (a) Positive incorporation (A-Lis, C,G,T, polymerase). (b) DNA polymerase withheld (ALis,C,G,T). (c) Fluorescent nucleotide and DNA polymerase withheld (A,C,G,T). tion of background from nonspecific binding of lissamine-labeled nucleotides and other fluorescent impurities is less than $5 \%$ of the total number of objects. Hence, over $95 \%$ of the observed objects in Figure 1a are single molecules of DNA. Thus, it is possible to observe incorporation of fluorescently labeled nucleotides into single-DNA template molecules.

In conclusion, we have developed a polyelectrolyte multilayer-based optimized robust surface chemistry for anchoring DNA to glass surfaces. This technique utilizes electrostatic repulsion to achieve a very low background for fluorescence studies and to promote DNA polymerase activity on the surface. Thus, this surface-chemistry platform may be useful for single-molecule fluorescence studies of the various enzymes that interact with DNA and indeed for any case in which substrate molecules have a net charge.

\section{REFERENCES}

1.Weiss, S. 1999. Fluorescence microscopy of single biomolecules. Science 283:1676-1683.

2.Ha, T., I. Rasnik, H.P. Babcock, G.H. Gauss, T.M. Lohman, and S. Chu. 2002. Initiation and re-initiation of DNA unwinding by the Escherichia coli Rep helicase. Nature 419:638-641.

3.Ha, T., A.Y. Ting, J. Liang, W.B. Caldwell, A.A. Deniz, D.S. Chemla, P.G. Schultz, and S. Weiss. 1999. Single-molecule fluorescence spectroscopy of enzyme conformational dynamics and cleavage mechanism. Proc. Natl. Acad. Sci. USA 96:893-898.

4.Zhuang, X., L.E. Bartley, H.P. Babcock, R. Russell, T. Ha, D. Herschlag, and S. Chu. 2000. A single-molecule study of RNA catalysis and folding. Science 288:2048-2051.

5.Lu, H.P., L. Xun, and X.S. Xie. 1998. Singlemolecule enzymatic dynamics. Science 282:1877-1882.

6.Decher, G. 1997. Fuzzy nanoassemblies: toward layered polymeric multicomposites. Science 277:1232-1237.

7.Decher, G., Y. Lvov, and J. Schmitt. 1994. Proof of multilayer structural organization in self-assembled polycation polyanion molecular films. Thin Solid Films 244:772-777.

8.Lvov, Y., G. Decher, and G. Sukhorukov. 1993. Assembly of thin-films by means of successive deposition of alternative layers of DNA and poly(allylamine). Macromolecules 26:5396-5399

9.Lvov, Y., H. Haas, G. Decher, H. Mohwald, A. Mikhailov, B. Mtchedlishvily, E. Morgunova, and B. Vainstein. 1994. Successive deposition of alternative layers of polyelectrolytes and a charged virus. Langmuir 10:4232-4236.
10.Cassier, T., K. Lowack, and G. Decher 1998. Layer-by-layer assembled protein/polymer hybrid films: nanoconstruction via specific recognition. Supramol. Sci. 5:309-315.

11.Ladam, G., P. Schaaf, F.J.G. Cuisinier, G Decher, and J.C. Voegel. 2001. Protein adsorption onto auto-assembled polyelectrolyte films. Langmuir 17:878-882.

12.Chluba, J., J.C. Voegel, G. Decher, P. Erbacher, P. Schaaf, and J. Ogier. 2001. Peptide hormone covalently bound to polyelectrolytes and embedded into multilayer architectures conserving full biological activity. Biomacromolecules 2:800-805.

13.Stroock, A.D., M. Weck, D.T. Chiu, W.T.S. Huck, P.J.A. Kenis, R.F. Ismagilov, and G.M. Whitesides. 2000. Patterning electroosmotic flow with patterned surface charge. Phys. Rev. Lett. 84:3314-3317.

14.Unger, M.A., E.P. Kartalov, C.S. Chiu, H.A. Lester, and S.R. Quake. 1999. Single-molecule fluorescence observed with mercury lamp illumination. BioTechniques 27:10081014

15.Chiu, C.S., E.P. Kartalov, M.A. Unger, S.R Quake, and H.A. Lester. 2001. Single-molecule measurements calibrate green fluorescent protein surface densities on transparent beads for use with 'knock-in' animals and other expression systems. J. Neurosci. Methods 105:55-63.

This work was supported by National Institutes of Health grant nos. HG01642 and 5 T32GM07616 to S.R.Q. Address correspondence to Dr. Stephen $R$. Quake, Department of Applied Physics, MC128-95, Caltech, Pasadena, CA 91125, USA. email:quake@caltech.edu

Received 15 October 2002; accepted 2 January 2003.

\section{Emil P. Kartalov, Marc}

A. Unger, and Stephen

R. Quake

Caltech

Pasadena, CA, USA 